\title{
Oxygen use in chronic heart failure to relieve breathlessness: A systematic review
}

\author{
Reiko Asano ${ }^{1}$ - Stephen C. Mathai ${ }^{2}$ - Peter S. Macdonald ${ }^{3}$ - Phillip J. Newton ${ }^{4}$. David C. Currow ${ }^{5}$. Jane Phillips ${ }^{5}$. \\ Wing-Fai Yeung ${ }^{6} \cdot$ Patricia M. Davidson ${ }^{1}$
}

(C) Springer Science+Business Media, LLC, part of Springer Nature 2019

\begin{abstract}
To appraise published studies on the use of supplemental oxygen in chronic heart failure. Chronic breathlessness is a characterizing symptom of symptomatic heart failure resulting in substantial disability and healthcare utilization and is the primary reason for emergency room visits and hospitalizations. In spite of the variable evidence, oxygen therapy is commonly administered both acutely and chronically. Moreover, the role of oxygen therapy to relieve chronic breathlessness in heart failure is not well described, particularly in normoxemic or mild or intermittent hypoxemic states. In fact, several studies have shown the detrimental effects of oxygen therapy with normal oxygen saturation levels. A systematic review using PRISMA guidelines. Four databases PubMed, Embase, CINAHL, and Web of Science were systematically searched from January 2001 to January 2019 investigating the use of oxygen in heart failure. Duplicate articles were removed from the review. Titles and abstracts were screened for inclusion and exclusion criteria. The remaining full-text articles were reviewed and hand-searched for additional references. The quality of the full-text articles was assessed using standardized critical appraisal instruments by the Joanna Briggs Institute. A total of 11 studies, including three intervention and eight non-interventions studies, were included in this review from 1072 nonduplicated records retrieved. Sample size ranged from 4 to 5862. In spite of common usage, this review suggests that there are scant data available to justify the use of oxygen in individuals with non-hypoxemic chronic heart failure and chronic breathlessness.
\end{abstract}

Keywords Oxygen use $\cdot$ Oxygen therapy $\cdot$ Breathlessness $\cdot$ Heart failure

Electronic supplementary material The online version of this article (https://doi.org/10.1007/s10741-019-09814-0) contains supplementary material, which is available to authorized users.

Reiko Asano

rasano1@jhu.edu

1 Johns Hopkins University School of Nursing, 525 N. Wolfe Street, Baltimore, MD 21205, USA

2 Johns Hopkins University School of Medicine, Baltimore, MD, USA

3 St. Vincent's Hospital Sydney, Sydney, Australia

4 Western Sydney University School of Nursing and Midwifery, Sydney, Australia

5 Faculty of Health, University of Technology Sydney, Sydney, Australia

6 The Hong Kong Polytechnic University School of Nursing, Hung Hom, Kowloon, Hong Kong, The Hong Kong SAR, China

\section{Introduction}

Heart failure is a condition where the heart is unable to fill or pump adequate blood to supply body's demand and is a lifethreatening chronic illness and the final pathway of many cardiovascular conditions [1]. Heart failure affects over 26 million people globally and the prevalence of heart failure is growing rapidly in both developed and developing countries worldwide [2]. It is a global health problem and the leading cause of death. In the USA, nearly 5.7 million adults are living with heart failure and nearly half of those die within 5 years of diagnosis [3].

Chronic breathlessness is a sentinel symptom of chronic heart failure affecting $50-70 \%$ of individuals and known to increase in intensity as disease progresses and particularly towards the end of life [4]. High symptom burden, disability, and reduced quality of life are common in both acute and chronic heart failure [4]. Chronic breathlessness is the sensation or perception of shortness of breath and a leading cause of emergency room visits and hospitalization in heart failure [5]. 
It is also both the experienced sensation as well as the reaction to adverse stimuli [6] and has been defined as "a chronic syndrome that persists despite optimal treatment of the underlying pathophysiology and that results in disability" [7]. Treatment approaches for chronic breathlessness vary with sparse evidence to inform treatment [8]. In many conditions, patients' prognosis may be better predicted by severity of breathlessness rather than physiological measures [6].

Oxygen therapy has been commonly prescribed to manage breathlessness in heart failure acutely and chronically in spite of poorly defined efficacy [5]. Although some oxygen-use studies have shown reductions in the intensity of breathlessness in patients with acute heart failure [9], currently there are no studies demonstrating the effectiveness and efficacy of long-term oxygen therapy in chronic heart failure for the symptomatic reduction of chronic breathlessness [5]. Moreover, there is a lack of research defining whether having an access to oxygen as needed (PRN) at home would reduce the need for hospital admissions for people with chronic breathlessness [5]. While oxygen therapy is commonly used to alleviate suffering from hypoxia with breathlessness in chronic heart failure, there is no evidence to inform the use of oxygen in chronic heart failure patients with chronic breathlessness alone [5]. Additionally, several studies have shown that hyperoxia can cause vasoconstriction in the cerebral, coronary, and systemic vasculature [10], and there are similar controversies regarding the use of oxygen in acute heart failure (AHF) [9]. Previous studies have concluded that excessive oxygen use could be detrimental to cardiac function with reduced stroke volume and cardiac output [9]. Understanding the role of oxygen to relieve breathlessness is predicated on an understanding of the pathophysiology and management of acute and chronic breathlessness. As heart failure management moves from the hospital to the community and hospital avoidance is a high priority, this is of increasing importance.

\section{Pathophysiology of chronic breathlessness}

Chronic breathlessness is caused by multiple factors and symptoms vary by intensity, unpleasantness, and a person's functional and emotional response [11]. This subjective symptom of shortness of breath often experienced by patients accounts for approximately half of tertiary hospital admissions for all patients [12]. The aetiology of breathlessness can be a mix of physiological, pathological, psychological, social, and environmental factors [13]. Breathlessness occurs most commonly due to compromised respiratory or cardiovascular systems, along with metabolic disorders, psychosocial conditions, and neuromuscular disorders [12]. More importantly, $90 \%$ of breathlessness is caused by heart failure, myocardial ischemia, chronic obstructive pulmonary disease (COPD), pneumonia, asthma, and psychological disorders [12]. Biopsychosocial causes of breathlessness include pain, distress, psychosocial problems, anger, panic attacks, and anxiety [13]. These conditions are known to increase respiratory work, air hunger, urge/need to breathe, or tightness caused by mismatch of pulmonary ventilation and the respiratory drive to breathe increases [13]. When breathlessness occurs, afferent receptors in the airways, chest and lung wall structures, and central respiratory motor activity are not matching [12].

Afferent and efferent receptors and central nervous system structures are involved in the development of breathlessness [13]. Afferent receptors are found in the airways, chest, and lung wall structures and are chemoreceptors that sense changes in the arterial blood $\mathrm{pH}$, carbon dioxide, and oxygen saturation [13]. The central chemoreceptors sense the changes in arterial blood $\mathrm{pH}$ and $\mathrm{CO}_{2}$ [13]. Chemoreceptors in the carotid bodies receive information from medulla about the blood gas levels of oxygen $\left(\mathrm{O}_{2}\right)$, carbon dioxide $\left(\mathrm{CO}_{2}\right)$, and hydrogen $\left(\mathrm{H}^{+}\right)$[12]. When $\mathrm{CO}_{2}$ increases, chemoreceptors are stimulated to increase the respiratory motor activity [13]. The build-up of $\mathrm{H}^{+}$lowers the $\mathrm{pH}$ level in body, known as respiratory acidosis. Efferent signals descend to the diaphragm and when the central processing in the brain senses a mismatch between the afferent and efferent signals, it results in breathlessness [12]. In other words, breathlessness occurs when the need for ventilation (afferent signals) and physical breathing (efferent signals) is not matched [12]. The job of afferent receptors is to assess if the efferent is signalling to the ventilatory muscle effectively and the required demands such as air pressure, air flow, and lung movement are being met [12]. When they are not responding to each other appropriately, the intensity of the breathlessness increases [12]. The breathlessness and conscious sensation of muscular effort are the result of motor signals being sent to the chest wall while the sensory cortex is activated [13].

\section{Breathlessness and anxiety cycle}

Understanding the relationship between anxiety and acute breathlessness is important in considering the use of oxygen therapy, particularly in the context of socialized response [14]. Likely in many healthcare encounters when an exacerbation of chronic breathlessness acutely worsens, oxygen is administered. The respiratory sensations are influenced by neural processing, occurring in the insular cortex, anterior cingulate cortex, and amygdala of the brain $[15,16]$. While this exact same area of the brain controls pain, anxiety, anticipation of breathlessness, fear of breathlessness, and sense of inability to control the breathlessness, it is also correlated with increased brain activations of anticipation and perception of induced breathlessness [16]. Several previous studies on breathlessness and neuroimaging have shown the relationship between perception of breathlessness and emotional processing [15]. The perception of breathlessness is caused by two primary components: the sensory (intensity) and affective 
(unpleasantness) components [17]. Then, it is followed by secondary component: cognitive affective, such as emotional responses (suffering), which affects breathlessness related behavior in the future [17]. Moreover, the perception of breathlessness is influenced by physiology, pathophysiology, and a complex interplay of mood, anxiety, emotional disposition, and anticipation resulting in a cycle of breathlessness and anxiety experiences [17]. It is also not unrealistic to suggest that individuals with heart failure who have had multiple interactions with the health system anticipate oxygen therapy in breathlessness. In fact, Sepehrvand and colleague comment that "oxygen therapy remains a cornerstone of acute heart failure treatment in practice, but guidelines provide variable recommendations on its appropriate use." This underscores the expectations of patients when breathless [9].

In a more recent study, Booth et al. emphasize the importance of understanding the clinical breathlessness model consists of breathing, thinking, and functioning [6]. While a patient is experiencing breathlessness, there are three factors driving the vicious cycle of breathlessness. When a patient is experiencing breathlessness, it is compensated by the use of accessory muscles as along with increased respiratory rate, which leads to thoughts and fear of dying along with sense of panic and increased anxiety level, which then lead to decreased physical activities as a natural response to reserving more energy [6].

\section{Current management strategies for chronic breathlessness}

There are three main breathlessness management strategies that are used in clinical practice, particularly in chronic states: nonpharmacological approaches including pulmonary rehabilitation, supplemental oxygen, and pharmacological therapies. Combining pharmacological and non-pharmacological interventions, such as regular oral low-dose extended release morphine and pulmonary rehabilitation, has been shown to be effective in managing chronic breathlessness among advanced chronic obstructive pulmonary disease patients [11]. There is also an increasing number of breathlessness clinics that recognize the complex and multifaceted nature of this symptom. The majority of advanced heart failure management occurs at home and community settings, which involves patients and their carers. It is essential to integrate self-care plan, knowledge, and strategies in individual's daily chronic heart failure care [18].

Supplemental oxygen is often used for chronically hypoxemic patients. Supplemental oxygen works by changing chemoreceptor stimulation and breathing pattern; however, the effectiveness of relieving breathlessness varies [12]. A previous meta-analysis demonstrated that long-term oxygen therapy may improve survival in patients with severe hypoxemia [19]. However, when used with patients mild to moderate hypoxemia, it did not improve survival [19]. Moreover, the use of long-term supplemental oxygen of at least $15 \mathrm{~h}$ a day, including overnight for individuals with COPD with hypoxia at rest, has shown reduction in mortality after over 1 year of therapy [19]. Furthermore, ambulatory oxygen therapy (the use of oxygen during exercise and activities of daily living) has been used among patients with COPD with or without resting hypoxemia and has demonstrated improvement in exercise capacity and quality of life in fatigue domain [20]. Yet in heart failure, data is scarce.

Pharmacological therapy often entails using opioids. Short-term use of opioids reduces breathlessness, especially in advanced COPD, other lung disease, and cancer patients [12]. While opioids have been commonly prescribed to treat COPD patients with chronic musculoskeletal pain and uncontrollable breathlessness, opioids can be harmful by causing hypoventilation and cough suppression which leads to reduced mucous clearance [21]. A previous study highlighted the increased risk of respiratory-related mortality and all-cause mortality when opioid is used among older adults with COPD [21]. L-menthol has been used in some clinical settings and has been shown to relieve exercise-induced dyspnea in healthy subjects [22]. Although the effect of the L-mentholderived cooling sensation has not been fully understood, it may be used to relieve breathlessness by stimulating olfactory temperature-sensitive sensory neurons [22]. Despite benzodiazepines being widely used to relieve breathlessness in advanced diseases, their propensity to cause drowsiness and rapid tolerance limits them to second- or third-line treatment, when other options have failed [23].

Pulmonary rehabilitation is often used in the management of chronic lung diseases [12]. Oftentimes, pulmonary rehabilitation programs include exercise, as well as education, dietary advice, psychological support, and assessment in some cases [24]. This reduces exertional breathlessness during exercise and improves exercise tolerance and activity level [25]. Among patients with COPD patients, pulmonary rehabilitation has demonstrated to relieve breathlessness and fatigue, enhance the patient's sense of control, and improve healthrelated quality of life [24]. Heart failure-specific cardiac rehabilitation also improves cardiovascular function.

Other non-pharmacological approaches such as directing cool/cold air on the face have shown to reduce breathlessness in healthy individuals [12]. Some patients feel subjective relief of breathlessness when sitting in front of a fan or an open window [22]. A randomized control study found a handheld fan blowing air to the face reduce intensity of breathlessness among individuals with advanced disease $[5,6]$. Yet, the mechanism of the effect of air therapy is still unknown [22].

\section{Use of oxygen therapy}

Oxygen therapy is variably prescribed to manage breathlessness in heart failure, partly because patients with severe lung 
disease who are hypoxemic at rest, with exertion, or during sleep benefit from long-term oxygen therapy, in which oxygen therapy can prolong survival [19]. The adherence rate of oxygen therapy prescription is generally lower than the clinical trials that inform the clinical practice due to its cumbersome nature and requirement for delivery systems that are often noisy and difficult to manage [5]. In addition, oxygen therapy may cause irritation to the nose and presents a social stigma for the patient [5]. For these reasons and others, oxygen therapy can be burdensome for patients and their families [5].

Breathlessness in heart failure is caused by complex cardiopulmonary interactions rather than cardiovascular factors alone [26] and the burden of comorbidities should also be underscored. Shortness of breath without objective signs of pulmonary congestion is a common early symptom of heart failure with preserved ejection fraction (HFpEF) and heart failure with reduced ejection fraction (HFrEF) [27]. Although a few oxygen-use studies have reported improvement in symptoms and intensity of breathlessness in patients with acute heart failure and hypoxia, there are no studies that support the safety and efficacy of long-term home oxygen therapy administered to chronic heart failure patients without hypoxia [5]. To date, there are a lack of data examining whether access to oxygen therapy at home would reduce the need for hospital admission [5]. Furthermore, a systematic review on the use of oxygen therapy to relieve breathlessness in chronic end-stage disease conducted by Cranston et al. underscored the inability to draw conclusions due to the small numbers of participants and limited numbers of research studies available [19]. Current research suggests that long-term oxygen therapy should not be used in patients with chronic heart failure due to the lack of evidence supporting the benefit in relieving breathlessness and also the absence of harm [5]. Although a few previous studies highlighted a positive relationship between duration of oxygen and improved symptoms, the mechanism of improved symptoms and the impact on prognosis remain unclear [5]. Yet in spite of this, oxygen administration is commonly undertaken in inpatient, outpatient, and community settings [5].

There are three main oxygen delivery systems that are used in current clinical practice: conventional oxygen therapy, high-flow nasal oxygen therapy, and non-invasive or invasive ventilation. This review pertains to the use of oxygen in the management of breathlessness in the absence of hypoxemia. The aim of this review was to identify existing literature describing the use of oxygen to relieve breathlessness in HF.

\section{Methods}

This systematic review adheres to the PRISMA reporting guidelines for systematic reviews to identify relevant peerreviewed published articles.

\section{Search methods}

The electronic databases PubMed, Embase, CINAHL, and Web of Science were searched from January 2001 to January 2019 under the supervision of a health librarian. Search criteria were created using the medical subject headings (MeSH) and non-MeSH search terms: "heart failure," "cardiac failure," "heart decompensation," "myocardial failure," "congestive heart failure," "oxygen inhalation therapy," "oxygen therapy," "oxygen use," "adult," and "adult patient," "dyspnea," and "breathlessness." In addition, a manual search of relevant literature and reference list was performed to identify applicable studies to this review.

\section{Inclusion and exclusion criteria}

Published studies were included if the review (1) included a heart failure population, (2) addressed the use of oxygen in chronic heart failure, and (3) included other chronic illnesses, if $25 \%$ of the included population had heart failure. Articles were excluded if the study focused on (1) pediatric populations, (2) left ventricular assist device (LVAD) or implantable cardiac defibrillator (ICD) populations, (3) sleep disordered and sleep apnea populations, (4) non-English publications, and (5) reviews, editorials, guidelines, or conference abstracts.

\section{Search outcome}

The search resulted in 1159 relevant citations and were imported into a web-based systematic review software program (Covidence®). Eighty-seven duplicates were removed, resulting in 1072 articles. Titles and abstracts were reviewed against inclusion and exclusion criteria, which resulted in the exclusion of 935 . The full text of the remaining 137 articles were assessed and 7 were excluded as non-English publications and another 119 articles were excluded because they either did not address heart failure population, focused on pediatric population, or were reviews, systematic reviews, letters to editor, guidelines, or conference abstract. A total of 11 articles were included in this review (Fig. 1).

\section{Data abstraction}

Data extraction from 11 articles were undertaken using a data extraction form, summarizing study design, sample characteristics, and summary of study findings (Table 1) [28]. Data were categorized as intervention studies and nonintervention studies, using the guidance on the conduct of narrative synthesis in systematic reviews [28]. 


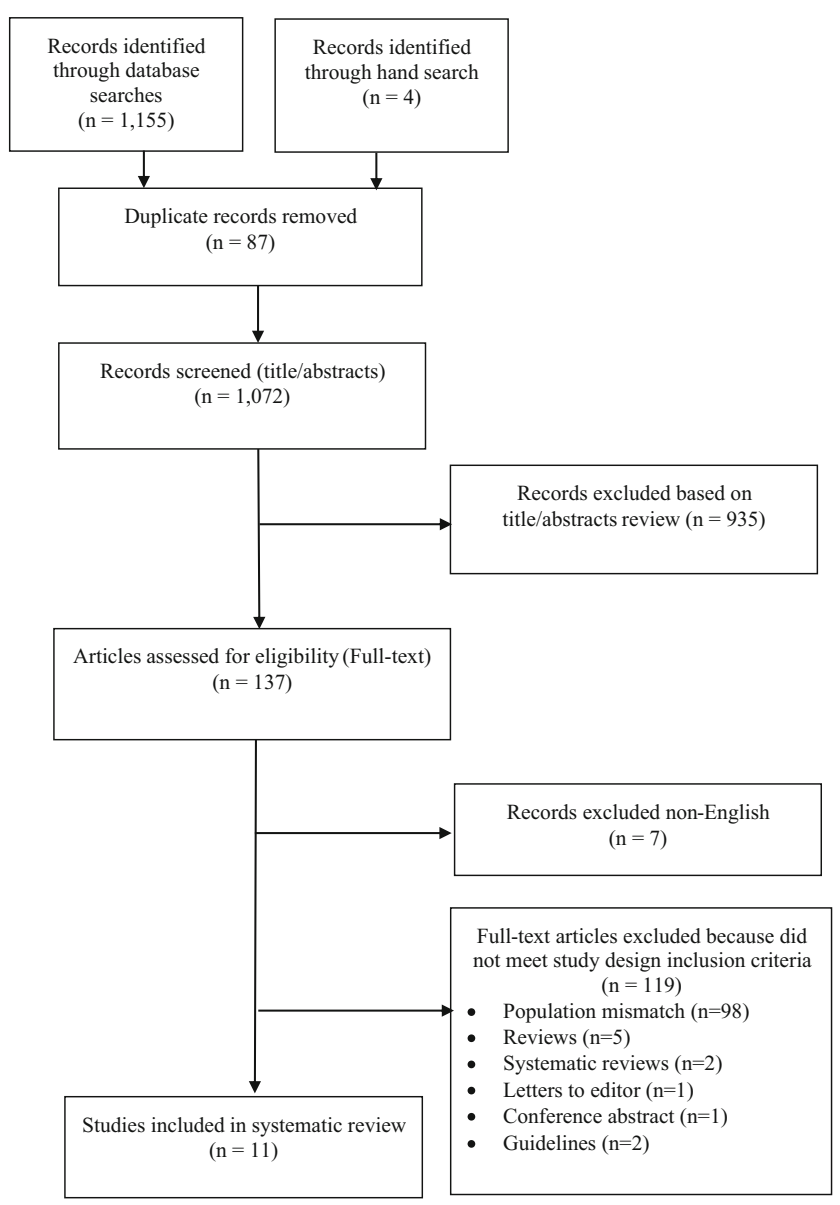

Fig. 1 Flowchart of included studies

\section{Results}

\section{Study characteristics}

The majority $(n=8)$ of the 11 eligible studies were nonintervention studies. Of the three intervention studies, there were two randomized control trials (RCTs) [5] and one experimental study [29]. Non-intervention studies included three observational studies [30]-32], one cohort study [33], two descriptive studies [34, 35], one case series [36], and one retrospective observational study [37]. Synthesized information on research design, sample size, and characteristics and findings is presented in Table 1. Studies were conducted in the USA, UK, Australia, Japan, and Europe (Turkey, Spain, France, and Netherlands). The majority of participants were male in all three intervention studies as majority of other heart failure study subjects are usually male. Most of the studies were undertaken in hospitals, several in outpatient centers, and a single study at home. Participants included New York Heart Association (NYHA) I-II [38], II-III [29, 35], III [36], and III-IV [5]. Ages ranged between 56 and 97 years old with the median age of 76.5 .
Intervention study findings

Long-term oxygen therapy In the inadequately powered RCT conducted in the UK, individuals with severe chronic heart failure (NYHA Class III-IV), recruited from the hospital outpatient clinics or the community (both urban and rural settings), were randomized ( $n=114$ ) to receive either long-term oxygen therapy prescribed for $15 \mathrm{~h}$ per day including overnight at approximately $28 \%$ oxygen while the control group $(n=51)$ received best medical therapy alone for 6 months [5]. This RCT showed no impact on quality of life measured by Minnesota Living with Heart Failure Questionnaire score at 6 months [5]. The quality of life score was lower among the long-term oxygen therapy group at 3 months [5]. Breathlessness scores improved in oxygen group at 3 months. However, there was no effect on 6-min walk test distance, Nterminal pro b-type natriuretic peptide (NTproBNP) level, and left ventricular ejection fraction in the oxygen group [5]. Although the survival rate was slightly better in the oxygen group, there was no statistically significant difference between two groups [5]. This RCT was significantly underpowered and only $11 \%$ of the participants adhered to oxygen as prescribed [5]. This RCT was stopped early by the funders due to poor patient adherence to home oxygen [5].

In an open label experimental study, 10 patients with NYHA classes II and III chronic heart failure received home oxygen for a minimum of $8 \mathrm{~h}$ at night at a flow rate of $4 \mathrm{~L} / \mathrm{min}$ via nasal cannula for 4 weeks [29]. After a month of nocturnal oxygen therapy, the functional status measured by the 6-min walk test improved clinically significantly by $53 \mathrm{~m}(17.8 \%$ increase) [29]. The quality of life measured by Minnesota Living with Heart Failure (MLWHF) also improved by 7 units (26.9\% increase) [29]. Considering the minimally significant clinical difference for MLWHF is 7.27 [39], the seven-unit improvement is moderately significant. However, there was no improvement in systolic and diastolic function with oxygen therapy or change in the baseline ejection fraction, stroke volume, or NTproBNP level with the oxygen therapy [29]. The authors concluded that although home oxygen therapy may be useful in chronic heart failure population, further randomized blinded studies are needed to confirm the findings and to determine the effects of oxygen use at night on mortality and re-hospitalization [29].

Effectiveness of acute oxygen/gas use In a phase III RCT conducted in an acute care hospital setting, 13 men with NYHA Class I-II heart failure and left ventricular systolic dysfunction were administered medical air or $40 \%$ oxygen at a flow rate of $10 \mathrm{~L} / \mathrm{min}$ for $15 \mathrm{~min}$, followed by highconcentration oxygen at flow rate of $15 \mathrm{~L} / \mathrm{min}$ via Hudson non-breathing mask for another $15 \mathrm{~min}$. The effects of oxygen use were complex [38]. Specifically, the supplemental oxygen decreased heart rate and cardiac output [38]. Moreover, 


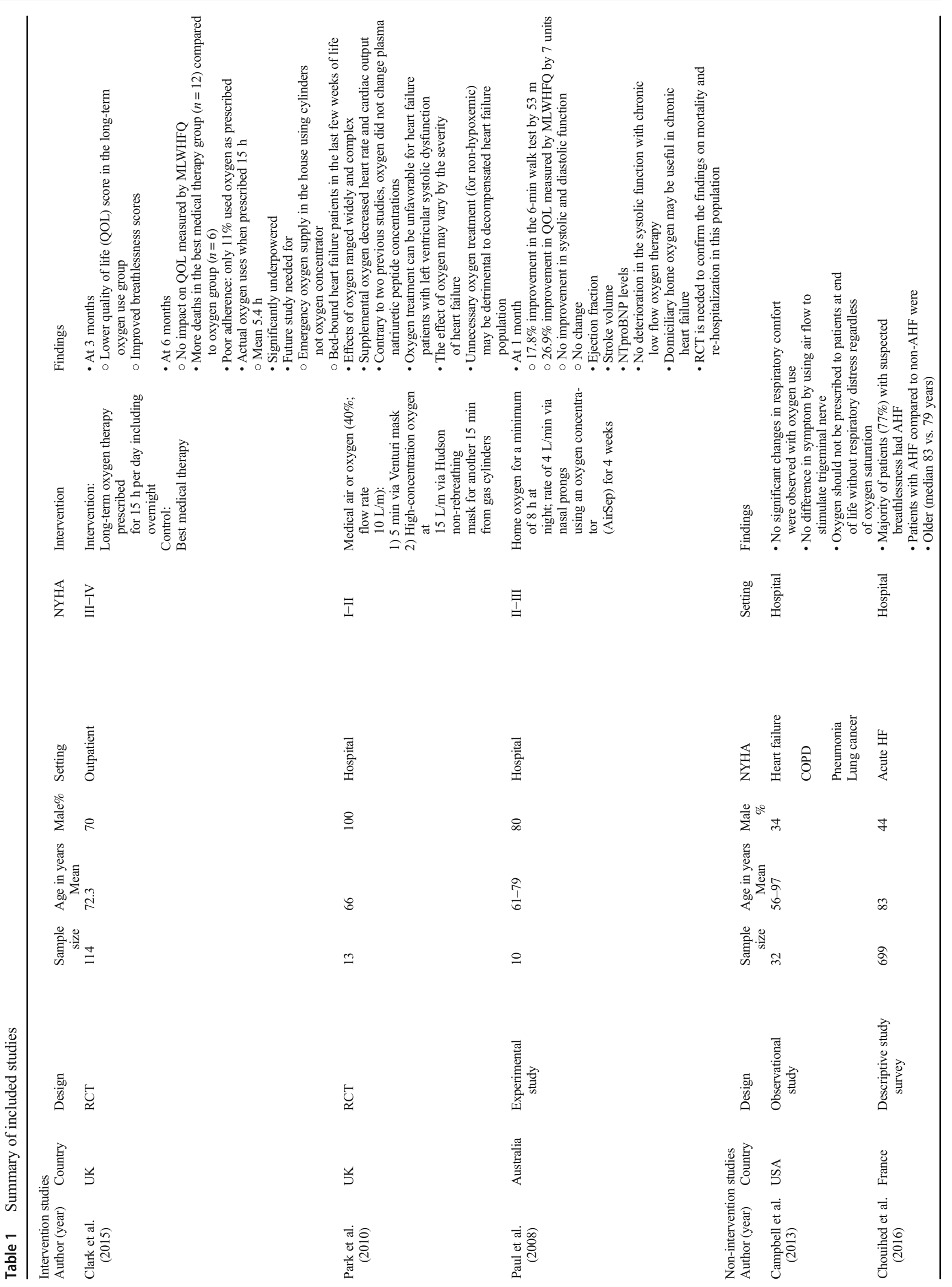




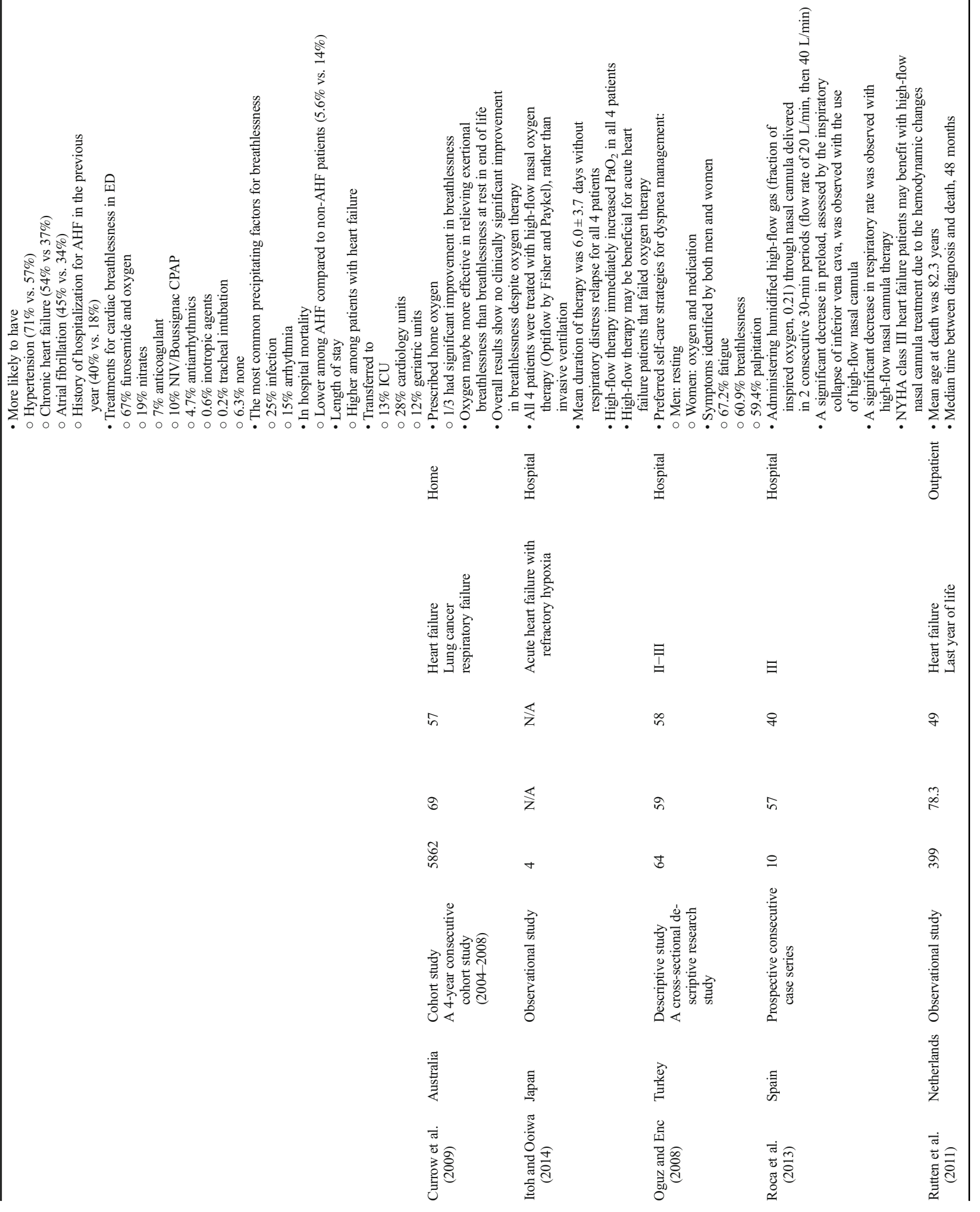



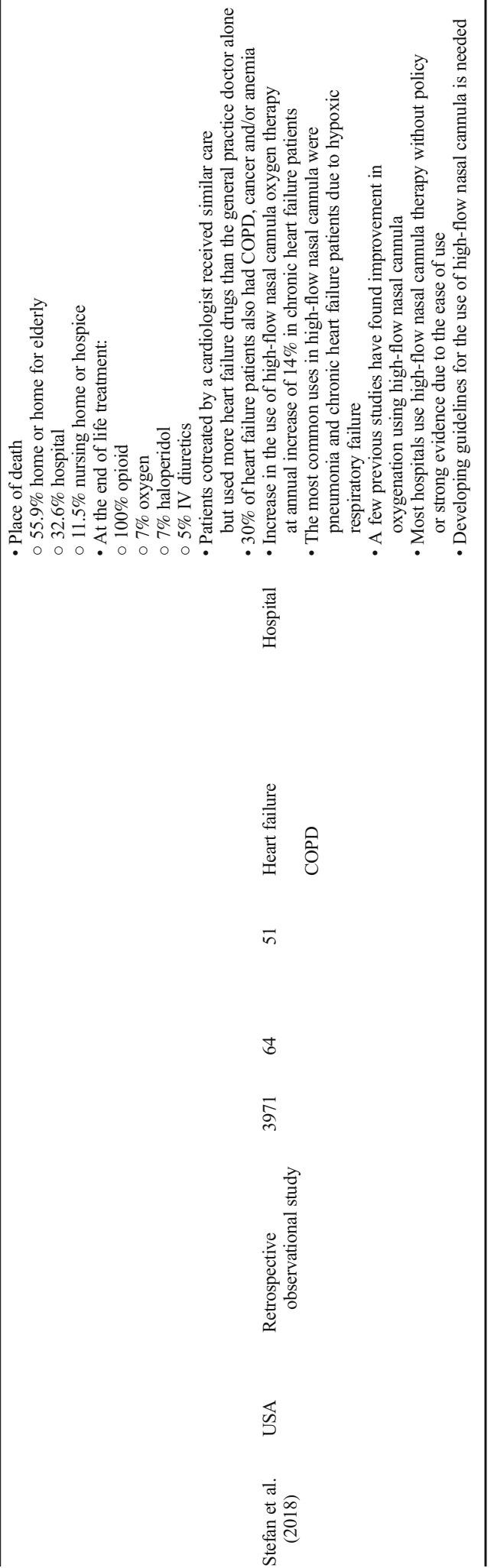

contrary to previous studies, oxygen did not change plasma natriuretic peptide concentrations [38]. The authors of this RCT concluded that the effect of oxygen may vary by the severity of heart failure and that unnecessary oxygen treatment (in the absence of hypoxemia) may be detrimental in patients with decompensated heart failure [38].

Patient's perception of oxygen use A qualitative sub-study of a long-term home oxygen therapy RCT revealed that participants did not adhere to oxygen therapy as prescribed, because they believed that oxygen use is for acute deterioration or for end of life [5]. Moreover, misunderstanding of the indication for oxygen use was making patients see long-term oxygen therapy as a burden rather than a benefit [5]. Many patients referred to oxygen therapy as an "altruistic act and as a way of accessing optimal clinical care" [5].

\section{Non-intervention study findings}

Out of eight eligible non-intervention studies, five of them specifically focused on heart failure (acute $(n=2)$ and chronic $(n=3)$ heart failure and the population mix of the other two studies included patients with heart failure and either lung cancer or respiratory failure. Out of all the non-intervention studies, two of them focused on patients at the end of life specifically [30, 32].

Oxygen use in breathlessness In a descriptive study using a nationwide French survey, DeFSSICA, out of 699 breathlessness patients with suspected AHF who were admitted to an emergency department, 2/3 were administered furosemide and oxygen for breathlessness [34]. In this study, majority of patients (77\%) with suspected breathlessness had AHF and that patients with AHF were older compared to non-AHF patients and $40 \%$ of them had history of hospitalization for AHF in the previous year [34]. In a cohort study conducted in Australia, $21 \%$ of the palliative care patients with chronic breathlessness were prescribed home oxygen and one-third of them had significant improvement in breathlessness; however, overall results showed no clinically significant improvement in breathlessness despite the oxygen administration [33]. The authors of this study highlighted that the role of oxygen remains unclear and there is a lack of studies to measure the impact of oxygen on quality of life and breathlessness in the heart failure population [33].

High-flow therapy A retrospective review of electronic medical record files reported an increase in the use of high-flow nasal cannula oxygen therapy with better survival among heart failure patients hospitalized with pneumonia [40]. The most common uses of high-flow nasal cannula were for pneumonia and chronic heart failure patients due to hypoxic respiratory failure [40]. In an observational study conducted in 
Japan, four acute heart failure patients with refractory hypoxia were treated with high-flow nasal oxygen therapy after failing conventional oxygen therapy [33]. Mean duration of this therapy was $6.0 \pm 3.7$ days without respiratory distress relapse for all four patients [33]. Although this study did not report the oxygen use during the course of $24 \mathrm{~h}$, the authors highlighted the benefit of high-flow therapy among acute heart failure patients with hypoxia who failed conventional oxygen therapy first [31]. Although previous studies have found improvement in oxygenation using high-flow oxygen [31, 40], most hospitals use high-flow nasal cannula therapy without policy [40]. In a study conducted in Spain, 10 patients with NYHA class III heart failure were administered humidified high-flow gas via nasal cannula, delivered in two consecutive $30-\mathrm{min}$ periods at a flow rate of $20 \mathrm{~L} / \mathrm{min}$, then increased to $40 \mathrm{~L} / \mathrm{min}$ [36]. A significant decrease in respiratory rate was observed in the participants suggesting that high-flow nasal cannula treatment may be beneficial in this population [36].

Gender preference While breathlessness was reported by $61 \%$ of the participants with NYHA class II and III chronic heart failure in a descriptive study conducted in Turkey, there were gender differences in terms of preferred breathlessness management [35]. While $65 \%$ of men preferred resting as breathlessness management, $81 \%$ of women preferred oxygen therapy and diuretics $(p=0.004)$ [35].

Oxygen use at end of life care Long-term oxygen therapy can be burdensome to both patients and caregivers as evidenced by adverse nasal effects, limited mobility, noisy compressors, cost, invaded space, and increased fire hazard [30]. While oxygen therapy can be useful with distressed or hypoxemic patients at the end of life, there is a lack of evidence to support the initiation or continuation of oxygen therapy among end of life heart failure patients who are not distressed or hypoxemic [30]. In a prospective nationwide survey in France, it was found that while all heart failure patients received opioid, only $7 \%$ of them received oxygen therapy [34].

\section{Discussion}

This systematic review highlights the lack of evidence for the use of oxygen in the HF population living with chronic breathlessness and some warnings about the potential dangers of using long-term oxygen therapy in this population. While RCTs included in this systematic review were both inadequately powered, RCTs generate the highest level of evidence. In both RCTs, the result of oxygen use was conflicting in terms of quality of life and relieving breathlessness. It was underscored that oxygen use may be detrimental when used in non-hypoxemic patients. The diversity and heterogeneity of study populations and outcome measures precluded meta- analysis. Moreover, only one study was undertaken among NYHA Class III and IV heart failure population on oxygen use. We also know that heart failure is a syndrome commonly associated with comorbidity and frailty and that breathlessness can be caused by deconditioning and other factors. Although the administration of oxygen therapy to relieve breathlessness in patients with heart failure is widely practiced, there is a lack of evidence to inform the use of oxygen in chronic heart failure. While a few oxygen-use studies have shown improvement in symptoms and intensity of breathlessness in acute heart failure, its safety and efficacy for chronic heart failure patients are understudied. Moreover, perception of breathlessness is heavily influenced by psychological and behavioral factors, such as anxiety, which is known to worsen future breathlessness. In order to provide optimal care that improves symptom management, quality of life, and prevents needless hospitalizations, better understanding of breathlessness management is needed. This will likely involve a more comprehensive understanding of not only the pathophysiology but the experience of breathlessness.

\section{Implications for future research}

This review has highlighted the importance of understanding the complexity of oxygen use from pathophysiology to psychosocial factors that may contribute to breathlessness symptoms. A recent systematic review in interstitial lung disease showed no effects of oxygen therapy on breathlessness during exercise, although exercise capacity was increased, underscoring the complexity of understanding mechanisms. Despite the common use of oxygen, future research is needed to investigate whether oxygen use in advanced heart failure population (NYHA III-IV) is useful or harmful and whether this therapeutic approach should be integrated with a comprehensive breathlessness management program to decrease symptom burden and improve quality of life.

\section{Implications for clinical practice}

This review underscores the conflicting results of oxygen use in heart failure population and the lack of evidence to inform guidelines in identifying the potential candidates that may benefit from oxygen administration, particularly in the palliative care setting.

\section{Limitations}

While we used a rigorous approach in systematically reviewing the existing literature, there may be a possibility we did not capture all relevant studies. Some studies did not have heart failure as their primary focus and detailed information may have been left out from their findings that may be relative to oxygen use in heart failure. This is particularly 
important in the context of multimorbidity and heart failure which is often a cardiogeriatric syndrome. There is also a lack of diversity as majority of participants were male. Furthermore, majority of the studies used convenient samples. These may limit the generalizability of the findings. In spite of these limitations, this systematic review highlights the current lack of evidence for oxygen use in heart failure population.

\section{Conclusion}

This review underscores the complexity of oxygen use in chronic heart failure populations. There are conflicting findings that oxygen use in heart failure may not be associated with improving quality of life or relieving breathlessness. In some cases, administration of oxygen may be harmful for this population. In spite of the common use of oxygen in chronic heart failure, a clear finding from this review is that there are scant data available and a lack of randomized clinical trials of oxygen use in heart failure populations. The fact is that only two studies were RCTs of varying quality and only one study was undertaken among NYHA Class III and IV heart failure populations on oxygen use which highlights a fertile ground for examining oxygen use in advanced heart failure populations.

\section{Compliance with ethical standards}

Conflict of interest No conflict of interest has been declared by the authors.

\section{Appendix}

("Oxygen use" OR "oxygen therapy") AND ("breathlessness" OR "dyspnea") AND (("Heart Failure"[Mesh] OR "cardiac failure" OR "heart decompensation" OR "heart failure" OR "myocardial failure" OR “chf” [tiab]))

\section{References}

1. Ponikowski P, Anker SD, AlHabib KF, Cowie MR, Force TL, Hu S, Jaarsma T, Krum H, Rastogi V, Rohde LE, Samal UC, Shimokawa H, Budi Siswanto B, Sliwa K, Filippatos G (2014) Heart failure: preventing disease and death worldwide. European Society of Cardiology Heart Failure 1(1):4-25

2. Savarese G, Lund LH (2017) Global public health burden of heart failure. Cardiac Failure Review 3(1):7-11

3. Center for Disease Control and Prevention (2016) Heart failure fact sheet, [Online]. Available: http://www.cdc.gov/dhdsp/data statistics/fact_sheets/fs_heart_failure.htm. Accessed 31 Mar 2019
4. Davidson PM, Macdonald P, Moser DK, Ang E, Paull G, Choucair S, Daly J, Gholizadeh L, Dracup K (2007) Cultural diversity in heart failure management: findings from the DISCOVER study (part 2). Contemp Nurse 25(1-2):50-61

5. Clark AL, Johnson M, Fairhurst C, Torgerson D, Cockayne S, Rodgers S, Griffin S, Allgar V, Jones L, Nabb S, Harvey I, Squire I, Murphy J, Greenstone M (2015) Does home oxygen therapy (HOT) in addition to standard care reduce disease severity and improve symptoms in people with chronic heart failure? A randomised trial of home oxygen therapy for patients with chronic heart failure. Health Technol Assess 19(75):1-120

6. Booth S, Ryan R, Spathis A (2016) Service delivery of complex interventions for refractory breathlessness. Curr Opin Support Palliat Care 10(3):228-235

7. Bausewein C, Schunk M, Schumacher P, Dittmer J, Bolzani A, Booth S (2018) Breathlessness services as a new model of support for patients with respiratory disease. Chron Respir Dis 15(1):48-59

8. Johnson MJ, Yorke J, Hansen-Flaschen J, Lansing R, Ekström M, Similowski T, Currow DC (2017) Towards an expert consensus to delineate a clinical syndrome of chronic breathlessness. Eur Respir J 49(5):1602277. https://doi.org/10.1183/13993003.02277-2016. Print

9. Sepehrvand N, Ezekowitz JA (2016) Oxygen therapy in patients with acute heart failure: friend or foe? Journal of American College of Cardiology: Heart Failure 4(10):783-790

10. Mak S, Azevedo ER, Liu PP, Newton GE (2001) Effect of hyperoxia on left ventricular function and filling pressures in patients with and without congestive heart failure. Chest 120(2):467473

11. Ahmadi Z, Currow DC, Ekstrom M (2017) Palliative oxygen for chronic breathlessness: what new evidence? Curr Opin Support Palliat Care 11(3):159-164

12. Coccia CB, Palkowski GH, Schweitzer B, Motsohi T, Ntusi NA (=2016) Dyspnoea: pathophysiology and a clinical approach. SAMJ: South African Medical Journal 106(1):32-36

13. Nishino T (2011) Dyspnoea: underlying mechanisms and treatment. Br J Anaesth 106(4):463-474

14. Breaden K, Collier A, Litster C, Allcroft P, Currow DC, Phillips JL (2019) Stigma and the in (visible) perspectives and expectations of home oxygen therapy among people with chronic breathlessness syndrome: a qualitative study. Palliat Med 33(1):82-90

15. Pattinson KT, Johnson MJ (2014) Neuroimaging of central breathlessness mechanisms. Curr Opin Support Palliat Care 8(3):225-233

16. Stoeckel MC, Esser RW, Gamer M, Buchel C, von Leupoldt A (2018) Dyspnea catastrophizing and neural activations during the anticipation and perception of dyspnea. Psychophysiology 55(4). https://doi.org/10.1111/psyp.13004

17. Hayen A, Herigstad M, Pattinson KT (2013) Understanding dyspnea as a complex individual experience. Maturitas 76(1):45-50

18. Riegel B, Moser DK, Anker SD, Appel LJ, Dunbar SB, Grady KL, Gurvitz MZ, Havranek EP, Lee CS, Lindenfeld J, Peterson PN, Pressler SJ, Schocken DD, Whellan DJ, American Heart Association Council on Cardiovascular Nursing, American Heart Association Council on Cardiovascular Nursing, American Heart Association Council on Clinical Cardiology, American Heart Association Council on Nutrition, Physical Activity, and Metabolism, American Heart Association Interdisciplinary Council on Quality of Care and Outcomes Research (2009) State of the science: promoting self-care in persons with heart failure: a scientific statement from the American Heart Association. Circulation 120(12):1141-1163

19. Cranston JM, Crockett A, Currow D (2008) Oxygen therapy for dyspnoea in adults. Cochrane Database Syst Rev (3)

20. Ameer F, Carson KV, Usmani ZA, Smith BJ (CD000238, 2014) Ambulatory oxygen for people with chronic obstructive pulmonary 
disease who are not hypoxaemic at rest. Cochrane Database Syst Rev 6

21. Vozoris NT, O’Donnell DE, Gill SS (2016) Opioids and adverse outcomes in elderly chronic obstructive pulmonary disease patients. Eur Respir J 48(6):1818-2016

22. Kanezaki M, Ebihara S (2017) Effect of the cooling sensation induced by olfactory stimulation by L-menthol on dyspnoea: a pilot study. Eur Respir J 49(4):1601823. https://doi.org/10.1183/ 13993003.01823-2016. Print

23. Simon ST, Higginson IJ, Booth S, Harding R, Weingärtner V, Bausewein C, Cochrane Pain, Palliative and Supportive Care Group (2016) Benzodiazepines for the relief of breathlessness in advanced malignant and non-malignant diseases in adults. Cochrane Database Syst Rev 2016(1):CD007354

24. McCarthy B, Casey D, Devane D, Murphy K, Murphy E, Lacasse Y (2015) Pulmonary rehabilitation for chronic obstructive pulmonary disease. Cochrane Database Syst Rev 2:CD003793

25. Coccia PF, Altman J, Bhatia S, Borinstein SC, Flynn J, George S, Goldsby R, Hayashi R, Huang MS, Johnson RH, Beaupin LK, Link MP, Oeffinger KC, Orr KM, Pappo AS, Reed D, Spraker HL, Thomas DA, von Mehren M, Wechsler DS, Whelan KF, Zebrack BJ, Sundar H, Shead DA (2012) Adolescent and young adult oncology clinical practice guidelines in oncology. JNCCN J Natl Compr Cancer Netw 10(9):1112-1150

26. Dube BP, Agostoni P, Laveneziana P (2016) Exertional dyspnoea in chronic heart failure: the role of the lung and respiratory mechanical factors. Eur Respir Rev 25(141):317-332

27. Kemp CD, Conte JV (2012) The pathophysiology of heart failure. Cardiovasc Pathol 21(5):365-371

28. Popay J, Roberts H, Sowden A, Petticrew M, Arai L, Rogers M, Britten N, Rosen K, Baldwin S, Duffy S (2003), Developing guidance on the conduct of narrative synthesis in systematic reviews. [Online]. Available: https://www.lancaster.ac.uk/shm/research/nssr/ research/dissemination/publications/medsoc sept 05.pdf

29. Paul B, Joseph M, De Pasquale CG (Jun. 2008) Domiciliary oxygen therapy improves sub-maximal exercise capacity and quality of life in chronic heart failure. Heart Lung Circ 17(3):220-223

30. Campbell ML, Yarandi H, Dove-Medows E (2013) Oxygen is nonbeneficial for most patients who are near death. J Pain Symptom Manag 45(3):517-523

31. Itoh T, Ooiwa H (2014) Successful treatment with high-flow nasal oxygen therapy in four patients with congestive heart failure resistance to conventional oxygen supply. J Card Fail 20(10): S151-S152

32. Rutten FH, Heddema WS, Daggelders GJA, Hoes AW (2012) Primary care patients with heart failure in the last year of their life. Fam Pract 29(1):36-42

33. Currow DC, Agar M, Smith J, Abernethy AP (2009) Does palliative home oxygen improve dyspnoea? A consecutive cohort study. Palliat Med 23(4):309-316

34. Chouihed T, Manzo-Silberman S, Peschanski N, Charpentier S, Elbaz M, Savary D, Bonnefoy-Cudraz E, Laribi S, Henry P, Girerd N, Zannad F, el Khoury C (2016) Management of suspected acute heart failure dyspnea in the emergency department: results from the French prospective multicenter DeFSSICA survey. Scand J Trauma Resusc Emerg Med 24(1):112

35. Oguz S, Enc N (2008) Symptoms and strategies in heart failure in Turkey. Int Nurs Rev 55(4):462-467

36. Roca O, Pérez-Terán P, Masclans JR, Pérez L, Galve E, Evangelista A, Rello J (2013) Patients with New York Heart Association class III heart failure may benefit with high flow nasal cannula supportive therapy: high flow nasal cannula in heart failure. J Crit Care 28(5): 741-746

37. Stefan MS, Eckert P, Tiru B, Friderici J, Lindenauer PK, Steingrub JS (2018) High flow nasal oxygen therapy utilization: 7-year experience at a community teaching hospital. Hosp Pract 46(2):73-76

38. Park JH, Balmain S, Berry C, Morton JJ, McMurray JJ (2010) Potentially detrimental cardiovascular effects of oxygen in patients with chronic left ventricular systolic dysfunction. Heart 96(7):533538

39. de Tejada MG-S et al (2019) Responsiveness and minimal clinically important difference of the Minnesota living with heart failure questionnaire. Health Qual Life Outcomes 17(1):32-36

40. Stefan MS, Priya A, Pekow PS, Lagu T, Steingrub JS, Hill NS, Nathanson BH, Lindenauer PK (2018) The comparative effectiveness of noninvasive and invasive ventilation in patients with pneumonia. J Crit Care 43:190-196

Publisher's note Springer Nature remains neutral with regard to jurisdictional claims in published maps and institutional affiliations. 